\title{
Should all hip and knee prosthetic joints be aspirated prior to revision surgery?
}

\author{
Femke Staphorst ${ }^{1}$ - Paul C. Jutte ${ }^{2} \cdot$ Alexander L. Boerboom $^{2} \cdot$ Greetje A. Kampinga $^{1}$ • Joris J. W. Ploegmakers ${ }^{2}$. \\ Marjan Wouthuyzen-Bakker ${ }^{1}$
}

Received: 18 June 2020 / Accepted: 11 January 2021 / Published online: 9 February 2021

(c) The Author(s) 2021

\begin{abstract}
Aims It is essential to exclude a periprosthetic joint infection (PJI) prior to revision surgery. It is recommended to routinely aspirate the joint before surgery. However, this may not be necessary in a subgroup of patients. The aim of our study was to investigate if specific clinical and implant characteristics could be identified to rule out a PJI prior to revision surgery.

Methods We retrospectively evaluated clinical and implant characteristics of patients who underwent a hip or knee revision surgery between October 2015 and October 2018. Patients were diagnosed with a PJI according to the MSIS diagnostic criteria.

Results A total of 156 patients were analyzed, including 107 implants that were revised because of prosthetic loosening and 49 because of mechanical failure (i.e. instability, malalignment or malpositioning). No PJI was diagnosed in the group with mechanical failure. In the prosthetic loosening group, 20 of 107 were diagnosed with a PJI (19\%). Although there was a significantly lower chance of having a PJI with an implant age of $>5$ years combined with a $\mathrm{CRP}<5 \mathrm{mg} / \mathrm{L}$, an infection was still present in 3 out of 39 cases (8\%).

Conclusion Implants with solely mechanical failure without signs of loosening and low inflammatory parameters probably do not require a synovial fluid aspiration. These results need to be confirmed in a larger cohort of patients. In case of prosthetic loosening, all joints need to be aspirated before surgery as no specific characteristic could be identified to rule out an infection.
\end{abstract}

Keywords Periprosthetic joint infection $\cdot$ Revision surgery $\cdot$ Aspiration $\cdot$ Aseptic loosening $\cdot$ Mechanical failure

\section{Introduction}

Total joint arthroplasty is one of the most commonly performed surgical procedures in orthopedics. Unfortunately, a prosthetic joint eventually requires revision, either due to mechanical failure (i.e. instability, malalignment or malpositioning) or loosening of the prosthesis [1,2]. Published records describe that around $10-20 \%$ of patients undergoing revision surgery have unexpected positive cultures during

Marjan Wouthuyzen-Bakker

m.wouthuyzen-bakker@umcg.nl

1 Department of Medical Microbiology and Infection Prevention, University of Groningen, University Medical Center Groningen, PO Box 30.001, 9700 RB Groningen, The Netherlands

2 Department of Orthopaedic Surgery, University of Groningen, University Medical Center Groningen, Groningen, The Netherlands revision [3-6], and these patients have a worse outcome compared with aseptic cases [7-10]. Therefore, it is critical to rule out a periprosthetic joint infection (PJI) prior to revision surgery because the revision of an infected prosthesis requires other surgical and antibiotic interventions than a revision for a non-infected prosthesis. Currently, there is no single test to confirm or exclude a PJI. Low serum inflammatory parameters such as C-reactive protein (CRP) and erythrocyte sedimentation rates (ESR) are false negative in a proportion of patients with a low-grade infection [11-14]. For this reason, it is advised not to rely on serum inflammatory parameters to rule out an infection [15]. Consequently, many experts recommend routine synovial fluid analysis prior to revision surgery. In particular, a synovial leucocyte count below 1500 cells $/ \mu \mathrm{L}$ and/or negative biomarkers (e.g. alpha defensin or calprotectin) have a very high negative predictive value and may be used in this regard [16-19]. However, it is known that specific clinical and implant characteristics increase the risk of having a PJI, such as revision prostheses 
and early prosthetic loosenings [20,21]. On the other hand, prostheses being revised for mechanical reasons may have a lower odd of having a PJI. Along these lines, identifying a subgroup of patients undergoing revision surgery in whom a synovial fluid aspiration is not necessary would be favorable. Therefore, the aim of our study was to investigate if specific clinical and implant characteristics could be identified to rule out a PJI and in whom a synovial fluid aspiration would be redundant.

\section{Materials and methods}

\section{Study design and inclusion criteria}

We performed a retrospective, observational cohort study in which the data of all patients who underwent a one or twostage revision of a total joint prosthesis were collected and evaluated (October 2015 until October 2018). The following inclusion criteria were applied:

1. Patients undergoing total revision surgery of a hip or knee prosthesis.

2. The revision surgery was performed more than 3 months after initial replacement.

Exclusion criteria:

1. Patients $<18$ years of age.

2. Patients with a fracture as an indication for revision.

3. Patients with missing intra-operative tissue cultures.

4. Patients who underwent revision surgery for acute PJI.

Patients were classified as having a PJI according to the Musculoskeletal Infection Society (MSIS) published in 2013 [22]. The indication for revision surgery was divided into two categories: prosthetic loosening or mechanical failure (i.e. instability, malalignment or malpositioning). The patient was assigned to the category prosthetic loosening when there was mechanical failure simultaneously with implant loosening diagnosed on X-ray and/or nuclear imaging (i.e. three-phase bone scintigraphy using ${ }^{99 \mathrm{~m}}$ technetiumhydroxymethylene diphosponate). The study was approved by the local Medical Ethical Committee (Reference Number: 201900356).

\section{Data collection}

The following clinical variables were collected: patients' medical history (including the presence of rheumatoid arthritis, the use of immunosuppressive drugs and/or antibiotics), specific characteristics of the prosthesis (cemented or uncemented, revised or primary, age of the prosthesis at the time of revision, etc.), presence of other osteosynthesis material (OSM) around the prosthesis (e.g. plates or screws), preoperative serum inflammatory markers (CRP and ESR) and details on intra-operative cultures.

\section{Handling of cultures}

During revision surgery, five intra-operative biopsies and one synovial fluid aspiration were obtained for culture. In addition, the prosthesis was vortexed for $30 \mathrm{~s}$ in Ringer lactate, sonicated for $1 \mathrm{~min}$ at $40.000 \mathrm{~Hz}$, and again vortexed for $30 \mathrm{~s}$. Subsequently, one hundred microliters of sonication fluid was plated on blood agar plates and $10 \mathrm{~mL}$ of sonication fluid was incubated in blood culture bottles (BD $\left.\mathrm{BACTEC}^{\mathrm{TM}}\right)$. Each sample and positive sonication fluid was cultured for 9-11 days on blood and chocolate agar under aerobic conditions (with $5 \% \mathrm{CO}_{2}$ ) and on Brucella blood agar under anaerobic conditions. In addition, all samples were cultured in fastidious broth. All broths were subcultured on blood and Brucella blood agar after 7 days of incubation. Subcultures were incubated for 2 days. The bacterial species was determined using the matrix-assisted laser desorption/ionization time-of-flight analyzer (MALDI-TOF).

\section{Statistical analysis}

All statistical analyses were performed using IBM SPSS statistic version $23^{\circledR}$. A Chi-square test (or a Fisher exact test when needed) was used to analyze the difference between the groups for categorical variables, and a one-way ANOVA (or Kruskal-Wallis test when data were not normally distributed) for continuous variables. To analyze the effect of possible risk factors for PJI, a logistic regression analysis was performed. Variables with a significance level of $<0.1$ were subsequently analyzed in a multivariate logistic regression model. The Kaplan-Meier curve was used to evaluate the event-free survival time (time between implantation and revision) for three different groups: aseptic loosening, PJI, and mechanical failure. A significance level of $0.05(a=5 \%$; bilateral) was considered for all analyses.

\section{Results}

\section{Incidence of $\mathrm{PJ}$ in cases revised for mechanical failure versus prosthetic loosening}

A total of 156 patients that underwent revision of a hip or knee prosthesis were included (Table 1). None of the patients were on antibiotic treatment at the time from revision. From the 156 patients, 107 had the preoperative diagnosis of prosthetic loosening (69\%), while 49 patients were diagnosed solely with mechanical failure $(31 \%)$. The 
Table 1 Patient characteristics

\begin{tabular}{|c|c|c|c|}
\hline & $\begin{array}{l}\text { Prosthetic loosening } \\
n=107\end{array}$ & $\begin{array}{l}\text { Mechanical failure } \\
n=49\end{array}$ & $P$ value \\
\hline \multicolumn{4}{|l|}{ Baseline characteristics } \\
\hline Male sex & $47(44)$ & $19(39)$ & 0.603 \\
\hline Age, years & $67.5(11.8)$ & $64.6(11.6)$ & 0.152 \\
\hline Body mass index $\left(\mathrm{kg} / \mathrm{m}^{2}\right)$ & $28.6(6.0)$ & $29.6(5.2)$ & 0.299 \\
\hline Smoking & $21(20)$ & $10(20)$ & 1.000 \\
\hline \multicolumn{4}{|l|}{ Comorbidity } \\
\hline Hypertension & $47(44)$ & $20(41)$ & 0.731 \\
\hline Ischemic heart disease & $13(12)$ & $9(18)$ & 0.327 \\
\hline Heart failure & $5(5)$ & $1(2)$ & 0.666 \\
\hline Diabetes mellitus & $11(10)$ & $6(12)$ & 0.784 \\
\hline Chronic obstructive pulmonary disease & $8(8)$ & $4(8)$ & 1.000 \\
\hline Chronic renal insufficiency & $3(3)$ & $1(2)$ & 1.000 \\
\hline Liver cirrhosis & $1(1)$ & $1(2)$ & 0.540 \\
\hline Malignancy ${ }^{\mathrm{b}}$ & $5(5)$ & $0(0)$ & 0.177 \\
\hline Malignancy in bone ${ }^{b}$ & $2(2)$ & $0(0)$ & 1.000 \\
\hline Rheumatoid arthritis & $9(9)$ & $2(4)$ & 0.504 \\
\hline Gout & $1(1)$ & $1(2)$ & 0.531 \\
\hline $\begin{array}{l}\text { Any previous surgery of the affected joint apart } \\
\text { from primary placement }\end{array}$ & $60(56)$ & $23(47)$ & 0.305 \\
\hline Previous infection joint & $15(14)$ & $2(4)$ & 0.095 \\
\hline Immunosuppressive medication & $9(8)$ & $3(6)$ & 0.754 \\
\hline \multicolumn{4}{|l|}{ Prosthesis } \\
\hline \multicolumn{4}{|l|}{ Joint } \\
\hline Hip & $56(52)$ & $5(10)$ & $0.000^{\mathrm{a}}$ \\
\hline Knee & $51(48)$ & $44(90)$ & $0.000^{\mathrm{a}}$ \\
\hline Cemented prosthesis & $53(68)$ & $28(70)$ & 1.000 \\
\hline \multicolumn{4}{|l|}{ Type of prosthesis } \\
\hline Revision prosthesis & $36(34)$ & $6(12)$ & $0.006^{\mathrm{a}}$ \\
\hline OSM around prosthesis ${ }^{\mathrm{c}}$ & $15(14)$ & $3(6)$ & 0.184 \\
\hline PJI & $20(19)$ & $0(0)$ & $0.000^{\mathrm{a}}$ \\
\hline
\end{tabular}

Nominal variables are depicted as $n(\%)$, continuous variables are depicted as mean (SD) or median (interquartile range) when not normally distributed

${ }^{\text {a }} P$ values $<0.05$ were considered statistically significant

${ }^{\mathrm{b}}$ Active malignancy at the time of revision

${ }^{\mathrm{c}}$ OSM osteosynthesis material around the prosthesis (e.g. screws, plates)

majority of the prostheses revised for mechanical failure were knees (44 of 49, 90\%). Loosened prostheses were revised to the same extent in both the joints (56 hips, 51 knees). Overall, 13\% (20 of 156) of patients were diagnosed with a PJI. All 20 PJIs were diagnosed in patients with loosening of the implant, which entailed 19\% (20 of 107) of the cohort of patients with prosthetic loosening. No PJIs were diagnosed in the group of patients with mechanical failure. $92 \%$ of patients that underwent revision surgery due to mechanical failure had a CRP $<10 \mathrm{mg} / \mathrm{L}$.

\section{Incidence of PJI according to clinical and implant characteristics}

To identify which implant characteristics are associated with a PJI, we first analyzed to what extent the age of the prosthesis was associated with the likelihood of having a PJI. Cases diagnosed with a PJI had a similar time to revision as the cases revised for a mechanical reason [mean time to revision 7.9 years (95\% CI 3.90-11.90) versus 6.5 years (95\% CI 4.61-8.43), respectively, $P=0.541$ 


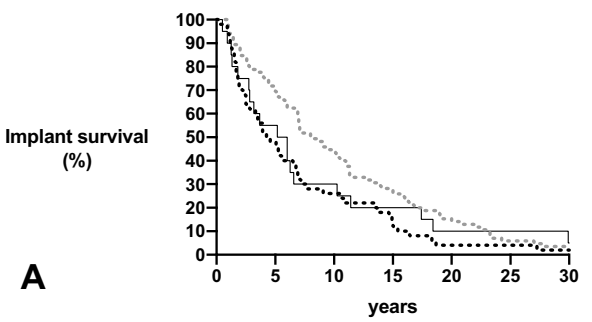

... Mechanical failure

A. Aseptic loosening

- PJI
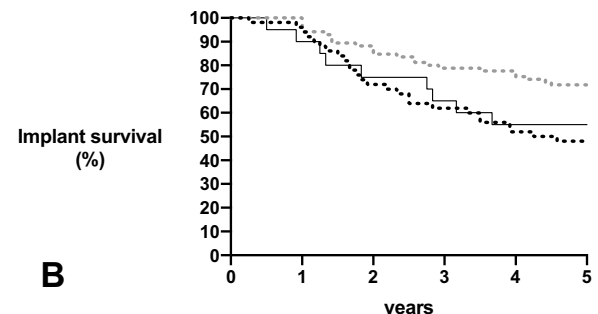

... Mechanical failure

- Aseptic loosening

- PJI

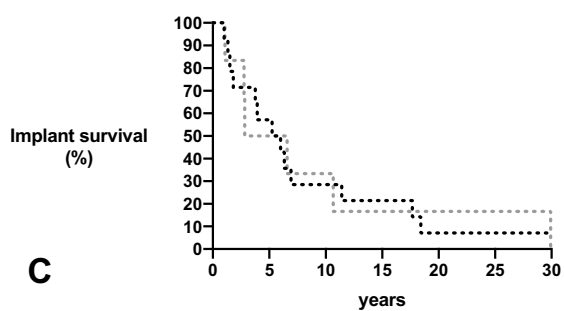

Fig. 1 Prosthesis age and implant survival. Kaplan-Meier depicting implant survival (time until revision) due to mechanical failure $(n=49)$, aseptic loosening $(n=87)$ or PJI $(n=20)$, depicted for
... PJI hip

.. PJI knee
(Fig. 1a, b)], but aseptic loosenings had a considerably longer time to revision [9.8 years, (95\% CI 8.08-11.43)]. These cases had the longest survival in the first 5 years with only $32 \%$ of cases ( 28 of 107 ) needing a revision within this time period. No differences in time to revision were observed in the PJI cases according to the type of joint or the type of prosthesis (Fig. 1c, d).

We additionally investigated if specific implant characteristics could differentiate between septic and aseptic loosening (Table 2). A previously treated infection

Table 2 Univariate and multivariate analyses for PJI in patients with loosening of the implant

\begin{tabular}{|c|c|c|c|c|c|c|}
\hline & $\begin{array}{l}\text { Septic loosen- } \\
\text { ing }(n=20)\end{array}$ & $\begin{array}{l}\text { Aseptic loos- } \\
\text { ening }(n=87)\end{array}$ & Unadjusted OR (95\% CI) & $P$ value & Adjusted OR (95\% CI) & $P$ value \\
\hline \multicolumn{7}{|l|}{ Implant characteristics } \\
\hline Previous surgery affected joint & $15(75)$ & $45(52)$ & $2.80(0.94-8.38)$ & $0.066^{*}$ & $2.14(0.47-9.71)$ & 0.327 \\
\hline Previous infection affected joint & $7(35)$ & $8(9)$ & $5.32(1.65-17.16)$ & $0.005^{*}$ & $2.82(0.74-10.75)$ & 0.129 \\
\hline Hip & $14(70)$ & $42(48)$ & $2.50(0.88-7.09)$ & $0.086^{*}$ & $2.70(0.85-8.54)$ & 0.091 \\
\hline Cemented prosthesis & $13(81)$ & $40(65)$ & $2.38(0.61-9.28)$ & 0.210 & & \\
\hline Revision prosthesis & $11(55)$ & $25(29)$ & $3.03(1.12-8.21)$ & $0.029^{*}$ & $1.21(0.29-5.08)$ & 0.794 \\
\hline OSM around prosthesis ${ }^{\mathrm{a}}$ & $4(20)$ & $11(13)$ & $1.71(0.48-6.04)$ & 0.409 & & \\
\hline \multicolumn{7}{|l|}{ Age of the prosthesis } \\
\hline$\leq 1$ year & $2(10)$ & $2(2)$ & $4.72(0.62-35.77)$ & 0.133 & & \\
\hline$\leq 2$ years & $5(25)$ & $11(13)$ & $2.30(0.70-7.60)$ & 0.171 & & \\
\hline$\leq 3$ years & $7(35)$ & $17(20)$ & $2.22(0.77-6.40)$ & 0.141 & & \\
\hline$\leq 4$ years & $9(45)$ & $21(24)$ & $2.57(0.94-7.05)$ & $0.066^{*}$ & $2.07(0.68-6.29)$ & 0.197 \\
\hline$\leq 5$ years & $9(45)$ & $28(32)$ & $1.72(0.64-4.64)$ & 0.280 & & \\
\hline$\leq 10$ years & $14(70)$ & $51(59)$ & $1.65(0.58-4.69)$ & 0.350 & & \\
\hline$\leq 15$ years & $16(80)$ & $62(71)$ & $1.61(0.49-5.30)$ & 0.431 & & \\
\hline$\leq 20$ years & $18(90)$ & 75 (86) & $1.44(0.30-7.01)$ & 0.652 & & \\
\hline
\end{tabular}

Nominal variables are depicted as $n(\%)$, continuous variables are depicted as mean (SD) or median (interquartile range) when not normally distributed

*Variables included in the multivariate binary logistic regression analysis

${ }^{\text {a } O S M}$ osteosynthesis material (e.g. screws or plates) 


\section{Aseptic loosening}

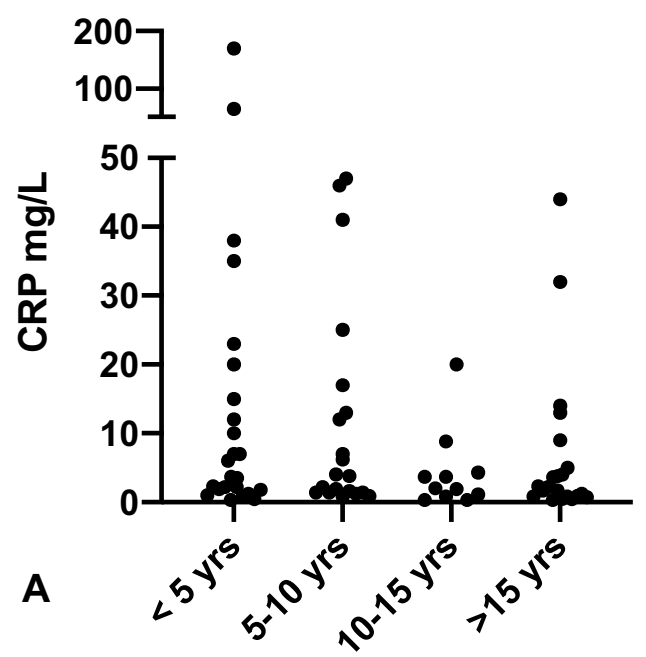

Aseptic loosening

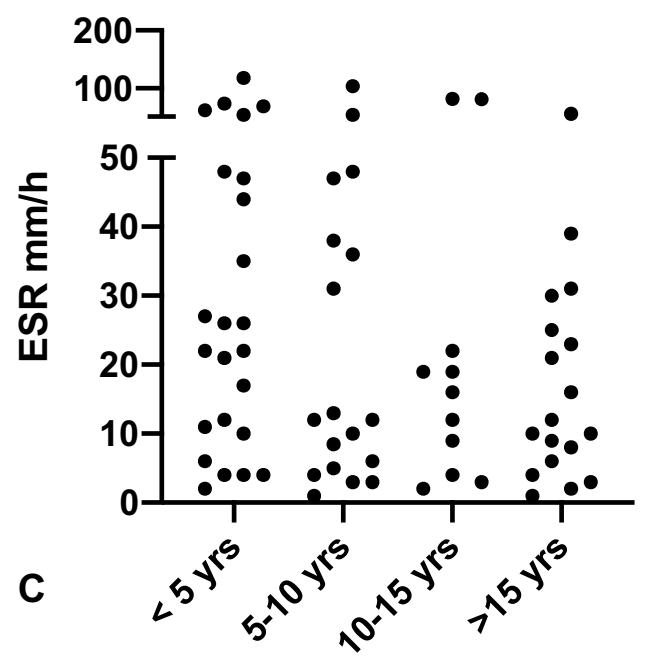

Fig. 2 Correlation between serum inflammatory parameters and prosthesis age at the time of revision. Scatterplot C-reactive protein (CRP) and erythrocyte sedimentation rate (ESR) of 76 patients with

of the affected joint and/or having a revision prosthesis were both significant risk factors for having a PJI in the univariate analysis, with an unadjusted OR of $5.32(95 \%$ CI $1.65-17.16, P=0.005)$ and 3.03 (95\% CI 1.12-8.21, $P=0.029)$, respectively. When testing for collinearity, 12 of the 36 patients with a revision prosthesis have had a previous infection of the affected joint. A hip prosthesis and/or having a prosthesis age of $\leq 5$ years were also associated with a higher odds of having a PJI, but these differences were not statistically significant. None of the analyzed variables were independent predictors for a PJI in the multivariate analysis (Table 2).

\section{Septic loosening}

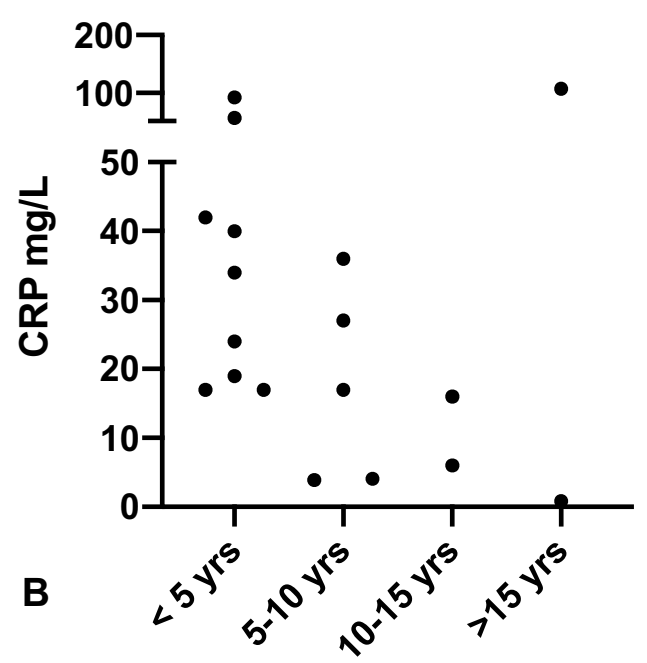

\section{Septic loosening}

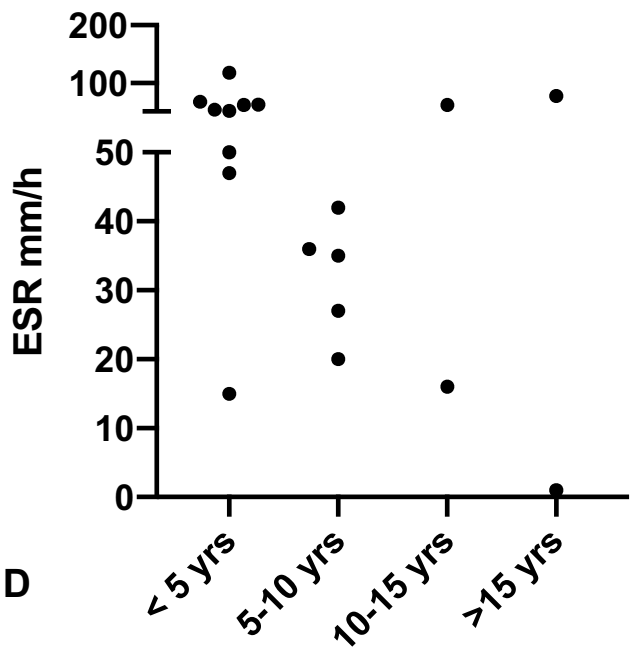

aseptic loosening $(\mathbf{a}, \mathbf{c})$ and 18 patients with septic loosening $(\mathbf{b}, \mathbf{d})$ (missing data in 11 and 2 and cases, respectively)

Patient characteristics (age, sex, smoking status, BMI, comorbidities and use of immune-suppressive drugs) were not associated with having a PJI (data not shown).

\section{Incidence of PJI in loosened implants and serum inflammatory markers}

We additionally analyzed whether a CRP $<5 \mathrm{mg} / \mathrm{L}$ and/ or an ESR $<30 \mathrm{~mm} / \mathrm{h}$ could rule out a PJI in patients with prosthetic loosening. Of the 20 patients with septic loosening, 3 patients had a CRP $<5 \mathrm{mg} / \mathrm{L}(15 \%)$. All of these three patients also had an ESR of $<30 \mathrm{~mm} / \mathrm{h}$. The causative 
microorganisms in these three cases were Staphylococcus epidermidis, Corynebacterium amycolatum and Staphylococcus lugdunensis, cultured in three, four and six intraoperative tissue samples, respectively. The patient with the $S$. lugdunensis infection had a sinus tract, which was probably the reason for the low CRP in this patient.

Figure 2 shows the serum CRP and ESR levels in relation to the age of the prosthesis at the time of revision. As depicted, a low serum CRP and/or ESR level could not rule out a PJI, even if the prosthesis was $>5$ years of age at the time of revision. The incidence of PJI in this group was $8 \%$ (3 out of 39). When excluding the patient with the sinus tract, the incidence was $5 \%$.

\section{Discussion}

In this retrospective observational cohort study, we analyzed whether specific clinical or implant characteristics could be identified to rule out a PJI prior to revision surgery without the need for synovial fluid aspiration. Our results indicate that patients who undergo revision surgery due to mechanical failure of the prosthesis probably do not need to be aspirated if accompanied with low-inflammatory parameters. We could not identify a subgroup of patients with loosening of the prosthesis in whom not aspirating the joint would be justifiable.

To our knowledge, we are the first who explicitly report on the incidence of PJI in prostheses with mechanical failure. A study by Khalid et al. did determine the incidence of unrecognized PJI in 72 patients undergoing revision for aseptic failure without any clinical suspicion of infection. These so-called aseptic failures were defined as the patients with implant instability and/or polyethylene wear but also included cases with implant loosening. Within this population, five patients turned out to have an unrecognized PJI (6.9\%), but it was not reported if the infections were only observed in the patients with prosthetic loosening [23]. The absence of infection in our patient population with mechanical failure should be interpreted with some caution. It should be noted that the gross majority of patients with mechanical failure in our analysis had low serum inflammatory markers. For this reason, we cannot conclude whether a synovial fluid aspiration is still needed in patients with an elevated CRP. In addition, our results need to be confirmed in a larger cohort of patients with merely mechanical failure, and a subanalysis on hips and knees should be performed. In our study, the majority of patients had mechanical failure of knees. As the aspiration of a hip joint is more complicated compared with knees, this group of patients is of particular interest.

Regarding patients with prosthetic loosening, the incidence of PJI in this particular group was high. The PJI incidence of $19 \%$ in patients with prosthetic loosening in our study is comparable with other reports [3-6]. We could not identify a subgroup of patients with prosthetic loosening in whom a PJI could be ruled out preoperatively without synovial fluid aspiration. Commonly described patient-related risk factors for a PJI such as male sex, diabetes, rheumatoid arthritis and obesity did not appear to be risk factors in our study [24-26]. A possible explanation might be that the importance of host-related factors are more pronounced when analyzing the risk of acute PJIs, rather than chronic ones. We did found that certain implant characteristics were associated with a higher odds of having a PJI. In accordance with previous studies [20,21, 27], early loosening occurred more often in the presence of a PJI. Portillo et al. demonstrated that in the first 2 years after implantation, revisions were performed more often for PJI (69\%) than for aseptic loosening (16\%). Indeed, in most studies, early loosening is defined as loosening within 2 years after implantation [20, 21]. In our study, the most prominent difference was observed using a cut-off of 4 years instead of 2 years. In addition, we identified that a previously treated infection of the affected joint and/or having a revision prosthesis are both associated with a higher odds of having a PJI. Despite the identification of these implant-related risk factors, PJIs were still detected in our population far after the 4-year implantation period. Moreover, even combined with a low serum CRP, a PJI could not be ruled out, not even when using a lower threshold of $5 \mathrm{mg} / \mathrm{L}$ instead of the accepted $10 \mathrm{mg} / \mathrm{L}$ used in the diagnostic criteria for PJI [22]. This finding again underlines that we cannot solely rely on serum CRP and ESR, as bacteria are able to remain in a 'dormant' and asymptomatic state within a biofilm without inducing any systemic inflammatory response [28, 29].

An important limitation of our study is the limited number of patients with a PJI $(n=20)$, which hampers the possibility to perform detailed subanalyses on high and low-risk groups for infection. Second, the preoperative diagnosis of loosening is challenging. In our cohort, next to conventional $\mathrm{X}$-rays to detect loosening, a bone scan was performed. This type of imaging may not be a routine practice in all hospitals and may result in false-positive scans when performed within 2 years after implantation for hips and within 5 years for knees. However, because the negative predictive value is high, we considered it as a valuable diagnostic tool to rule out infection [30]. Finally, we only studied CRP and ESR as serum inflammatory markers. There are other sensitive serum markers available, such as D-dimer and interleukin 6 (IL-6) that we have not incorporated in our daily clinical care [31,32]. Although these markers do not exhibit a diagnostic accuracy of $100 \%$ either, applying a combination of serum inflammatory markers may be beneficial $[33,34]$.

In conclusion, in case of prosthetic loosening, all joints need to be aspirated before revision surgery as no specific characteristic could be identified to $100 \%$ rule out an 
infection. A prosthetic implant that needs to be revised for a mechanical reason without any signs of loosening probably does not need a synovial fluid aspiration, in particular, not when combined with low serum inflammatory markers.

Open Access This article is licensed under a Creative Commons Attribution 4.0 International License, which permits use, sharing, adaptation, distribution and reproduction in any medium or format, as long as you give appropriate credit to the original author(s) and the source, provide a link to the Creative Commons licence, and indicate if changes were made. The images or other third party material in this article are included in the article's Creative Commons licence, unless indicated otherwise in a credit line to the material. If material is not included in the article's Creative Commons licence and your intended use is not permitted by statutory regulation or exceeds the permitted use, you will need to obtain permission directly from the copyright holder. To view a copy of this licence, visit http://creativecommons.org/licenses/by/4.0/.

\section{References}

1. Evans JT, Evans JP, Walker RW, Blom AW, Whitehouse MR, Sayers A (2019) How long does a hip replacement last? A systematic review and meta-analysis of case series and national registry reports with more than 15 years of follow-up. Lancet 393:647-654

2. Boyer B, Bordini B, Caputo D, Neri T, Stea S, Toni A (2019) What are the influencing factors on hip and knee arthroplasty survival? Prospective cohort study on 63619 arthroplasties. Orthop Traumatol Surg Res 105(7):1251-1256

3. Saleh A, Guirguis A, Klika AK, Johnson L, Higuera CA, Barsoum WK (2014) Unexpected positive intraoperative cultures in aseptic revision arthroplasty. J Arthroplasty 29:2181-2186

4. Ribera A, Morata L, Moranas J, Agulló JL, Martínez JC, Lopez Y et al (2014) Clinical and microbiological findings in prosthetic joint replacement due to aseptic loosening. J Inf Secur 69:235-243

5. Fernandez-Sampedro M, Salas-Venero C, Fariñas-Álvarez C, Sumillera M, Pérez-Carro L, Fakkas-Fernandez M et al (2015) 26 postoperative diagnosis and outcome in patients with revision arthroplasty for aseptic loosening. BMC Infect Dis 15:232

6. Purudappa PP, Sharma OP, Priyavadana S, Sambandam S, Villafuerte JA (2020) Unexpected positive intraoperative cultures (UPIC) in revision hip and knee arthroplasty - a review of the literature. $\mathrm{J}$ Orthop 17:1-6

7. Barrack RL, Aggarwal A, Burnett RSJ, Clohisy JC, Ghanem E, Sharkey P et al (2007) The fate of the unexpected positive intraoperative cultures after revision total knee arthroplasty. J Arthroplasty 22(6 Suppl 2):94-99

8. Padegimas EM, Lawrence C, Narzikul AC, Zmistowski BM, Abboud JA, Williams GR et al (2017) Future surgery after revision shoulder arthroplasty: the impact of unexpected positive cultures. J Shoulder Elbow Surg 26:975-981

9. Foruria AM, Fox TJ, Sperling JW, Cofield RH (2013) Clinical meaning of unexpected positive cultures (UPC) in revision shoulder arthroplasty. J Shoulder Elbow Surg. 22:620-627

10. Grosso MJ, Sabesan VJ, Ho JC, Ricchetti ET, Iannotti JP (2012) Reinfection rates after 1-stage revision shoulder arthroplasty for patients with unexpected positive intraoperative cultures. J Shoulder Elbow Surg 21:754-758

11. Piper KE, Fernandez-Sampedro M, Steckelberg KE, Mandrekar JN, Karau MJ, Steckelberg JM et al (2010) C-reactive protein, erythrocyte sedimentation rate and orthopedic implant infection. PLoS ONE 5:e9358

12. McArthur BA, Abdel MP, Taunton MJ, Osmon DR, Hanssen AD (2015) Seronegative infections in hip and knee arthroplasty: periprosthetic infections with normal erythrocyte sedimentation rate and C-reactive protein level. Bone Joint J 97:939-944

13. Johnson AJ, Zywiel MG, Stroh A, Marker DR, Mont MA (2011) Serological markers can lead to false negative diagnoses of periprosthetic infections following total knee arthroplasty. Int Orthop 35:1621-1626

14. Kheir MM, Tan TL, Shohat N, Foltz C, Parvizi J (2018) Routine diagnostic tests for periprosthetic joint infection demonstrate a high false negative rate and are influenced by the infecting organism. $\mathrm{J}$ Bone Joint Surg Am 100(23):2057-2065

15. Bauer TW, Bedair H, Creech JD, Deirmengian C, Eriksson H, Fillingham $Y$ et al (2019) Hip and knee section, diagnosis, laboratory tests: proceedings of international consensus on orthopedic infections. J Arthroplasty 34:S351-S359

16. Sousa R, Serrano P, Gomes Dias J, Oliveira JC, Oliveira A (2017) Improving the accuracy of synovial fluid analysis in the diagnosis of prosthetic joint infection with simple and inexpensive biomarkers: C-reactive protein and adenosine deaminase. Bone Joint J 99(3):351-357

17. Berger P, Van Cauter M, Driesen R, Neyt J, Cornu O, Bellemans J (2017) Diagnosis of prosthetic joint infection with alpha-defensin using a lateral flow device: a multicentre study. Bone Joint $\mathbf{J}$ 99(9):1176-1182

18. Wouthuyzen-Bakker M, Ploegmakers JJW, Ottink K, Kampinga GA, Wagenmakers-Huizenga JPC et al (2018) Synovial calprotectin: an inexpensive biomarker to exclude a chronic prosthetic joint infection. J Arthroplasty 33(4):1149-1153

19. De Vecchi E, Romano CL, De Grandi R, Cappelletti L, Villa F, Drago L (2018) Alpha defensin, leukocyte esterase, C-reactive protein, and leukocyte count in synovial fluid for pre-operative diagnosis of periprosthetic infection. Int J Immunopathol Pharmacol 32:2058738418806072

20. Portillo ME, Salvado M, Alier A, Sorli L, Martinez S, Horcajada JP et al (2013) Prosthesis failure within 2 years of implantation is highly predictive of infection. Clin Orthop Relat Res 471(11):3672-3678

21. Kokko MA, Abdel MP, Berry DJ, Butler RD, Van Citters DW (2019) A retrieval analysis perspective on revision for infection. Arthroplast Today 5(3):362-370

22. Parvizi J, Gehrke T (2014) International consensus group on periprosthetic joint infection definition of periprosthetic joint infection. J Arthroplasty 29:1331

23. Khalid V, Schonheyder HC, Nielsen PT, Kappel A, Thomsen TR, Aleksyniene R et al (2019) 72 revision surgeries for aseptic failure after hip or knee arthroplasty: a prospective study with an extended diagnostic algorithm. BMC Musculoskelet Disord 20(1):600

24. Blanco JF, Diaz A, Melchor FR, da Casa C, Pescador D (2019) Risk factors for periprosthetic joint infection after total knee arthroplasty. Arch Orthop Trauma Surg

25. Iqbal F, Shafiq B, Zamir M, Noor S, Memon N, Memon N, et al. (2019) Micro-organisms and risk factors associated with prosthetic joint infection following primary total knee replacement-our experience in Pakistan. Int Orthop

26. Arthroplasty Collaborative Mac TM (2019) Risk factors for periprosthetic joint infection following primary total hip arthroplasty: a 15-year, population-based cohort study. J Bone Joint Surg Am

27. Sharkey PF, Lichstein PM, Shen C, Tokarski AT, Parvizi J (2013) Why are total knee arthroplasties failing today-has anything changed after 10 years? J Arthroplasty 29(9):1774-1778

28. Davidson DJ, Spratt D, Liddle AD (2019) Implant materials and prosthetic joint infection: the battle with the biofilm. EFORT Open Rev 4(11):633-639 
29. Lebeaux D, Ghigo J-M, Beloin C (2014) Biofilm-related infections: bridging the gap between clinical management and fundamental aspects of recalcitrance toward antibiotics. Microbiol Mol Biol Rev 78(3):510-543

30. Sconfienza LM, Signore A, Cassar-Pullicino V, Cataldo MA, Gheysens O, Borens O et al (2019) Diagnosis of peripheral bone and prosthetic joint infections: overview on the consensus documents by the EANM, EBJIS, and ESR (with ESCMID endorsement). Eur Radiol 29(12):6425-6438

31. Shahi A, Kheir MM, Tarabichi M, Hosseinzadeh HRS, Tan TL, Parvizi J (2017) Serum D-Dimer test is promising for the diagnosis of periprosthetic joint infection and timing of reimplantation. J Bone Joint Surg Am 99(17):1419-1427

32. Yoon JR, Yang SH, Shin YS (2018) Diagnostic accuracy of interleukin-6 and procalcitonin in patients with periprosthetic joint infection: a systematic review and meta-analysis. Int Orthop 42(6):1213-1226

33. Huang J, Zhang Y, Wang Z, Dong Y, Zhao Y, Zheng J et al (2019) The serum level of D-Dimer is not suitable for distinguishing between prosthetic joint infection and aseptic loosening. J Orthop Surg Res 14(1):407

34. Qin L, Li F, Gong X, Wang J, Huang W, Hu N (2020) Combined measurement of D-Dimer and C-reactive protein levels: highly accurate for diagnosing chronic periprosthetic joint infection. J Arthroplasty 35(1):229-234

Publisher's Note Springer Nature remains neutral with regard to jurisdictional claims in published maps and institutional affiliations. 